\title{
Lie Group Classifications and Stability of Exact Solutions for Multidimensional Landau-Lifshitz Equations
}

\author{
Jiali Yu, Fuzhi Li, Hui Yang, Ganshan Yang* \\ School of Mathematics, Yunnan Normal University, Kunming, China \\ Email: jialiyu97@yahoo.com, lifuzhifight@foxmail.com,yh_m1026@aliyun.com.cn, \\ *yganshan@aliyun.com
}

Received 25 February 2016; accepted 25 April 2016; published 28 April 2016

Copyright (C) 2016 by authors and Scientific Research Publishing Inc.

This work is licensed under the Creative Commons Attribution International License (CC BY). http://creativecommons.org/licenses/by/4.0/

(c) (i) Open Access

\begin{abstract}
In this paper, based on classical Lie group method, we study multi-dimensional Landau-Lifshitz equation, and get its infinitesimal generator, symmetry group and new solutions. In particular, we build the connection between new exact solutions and old exact solutions. At the same time, we also prove that the initial boundary value condition of the three-dimensional Landau-Lifshitz equation admits a unique solution and discuss the stability of the solution.
\end{abstract}

Keywords

Lie Group, Multidimensional Landau-Lifshitz Equation, Explicit Solutions, Stability

\section{Introduction}

In 1935, the famous Landau-Lifshitz equations were proposed by Landau and Lifshitz [1] to describe the evolution of spin fields in continuum ferromagnet [2]. In this paper we study two important equations as follows

$$
\begin{gathered}
\mathrm{u}_{t}=\mathrm{u} \times \Delta \mathrm{u}, \\
\mathrm{u}_{t}=\lambda_{1} \mathrm{u} \times \Delta \mathrm{u}+\lambda_{2} \mathrm{u} \times \mathrm{u} \times \Delta \mathrm{u},
\end{gathered}
$$

where $\times$ denotes the vector cross-product in $\mathbb{R}^{3}, u=(u, v, w): \mathbb{R}^{n} \times \mathbb{R}^{+} \rightarrow \mathbb{R}^{3}$ is the spin density, $\lambda_{2}<0$ is a damping parameter. Emphasizing its parabolic character, (2) can also be considered as a quasilinear perturbation of the heat flow for harmonic maps by the (conservative) precession term $-\mathrm{u} \times \Delta \mathrm{u}$. The n-dimensional cylindrical symmetrical form of (1) is

${ }^{*}$ Corresponding author.

How to cite this paper: Yu, J.L., Li, F.Z., Yang, H. and Yang, G.S. (2016) Lie Group Classifications and Stability of Exact Solutions for Multidimensional Landau-Lifshitz Equations. Applied Mathematics, 7, 665-680.

http://dx.doi.org/10.4236/am.2016.77061 


$$
\mathrm{u}_{t}=\mathrm{u} \times \mathrm{u}_{r r}+\frac{n-1}{r} \mathrm{u} \times \mathrm{u}_{r}
$$

where $r=\sqrt{x_{1}^{2}+x_{2}^{2}+\cdots+x_{n}^{2}}$.

For the multidimensional case. Zhou and Guo proved the global existence of weak solution for the generalized Landau-Lifshitz equations at absence of Gilbert term [3]. Chang et al. considered the initial value problem for the 2-dimensional cylindrical symmetric Landau-Lifshitz equation without external magnetic field [4]. The soliton solutions to the Landau-Lifshitz equations with and without external magnetic field have been studied by many physicists and mathematicians [5]-[7]. For the Equation (3), when $n=2$, Guo and Yang have constructed an exact solution in unit sphere [8]. In [9] and [10], Guo and Han as well as Yang have also obtained an exact blow up solution for the n-dimensions form. In [11] and [12], Yang considered the relations between (1) and (2).

It is of great importance to find exact solutions of Landau-Lifshitz equations. But it is difficult to solve Landau-Lifshitz equations. As is known, the symmetry group technique is one of the powerful tools for solving a nonlinear differential equation (see [13]-[22]): the classical Lie group method [15] [16], the non-classical Lie group method [17] [18]. Xu and Liu have studied n-dimensional radial symmetric Landau-Lifshitz equation with external magnetic field in [19].

In this paper, the symmetry group of the n-dimensional Landau-Lifshitz equation is obtained by using the classical method in Section 2. The transformations leave the solutions invariant. In Section 3, we give the new solutions of Landau-Lifshitz equation from the known solutions [10]. Finally, the uniqueness and stability of the Landau-Lifshitz equation and the Landau-Lifshitz-Gilbert equation are given [20], respectively, in Section 4 and 5 .

\section{Lie Symmetry Group of the Landau-Lifshitz Equation}

Here are four independent variables $\mathrm{x}=(x, y, z)$ being spatial coordinates and $t$ the time, together with four dependent variables, the velocity field $\mathrm{u}=(u, v, w)$. In vector notation, the system has the form

$$
\left\{\begin{array}{l}
u_{t}=v \Delta w-w \Delta v, \\
v_{t}=w \Delta u-u \Delta w \\
w_{t}=u \Delta v-v \Delta u
\end{array}\right.
$$

According to the method of determining the infinitesimal generator of nonlinear partial differential equation [16], we take the infinitesimal generator of equation as follows:

$$
v=\xi \frac{\partial}{\partial x}+\eta \frac{\partial}{\partial y}+\varsigma \frac{\partial}{\partial z}+\tau \frac{\partial}{\partial t}+\varphi \varsigma \frac{\partial}{\partial u}+\psi \frac{\partial}{\partial v}+\chi \frac{\partial}{\partial w},
$$

where $\xi, \cdots, \chi$ are functions of $x, t, u,(1)$ is of second order $n=2$. Applying the first prolongation $p r^{(2)} \mathrm{v}$, we find

$$
\begin{gathered}
p r^{(1)} \mathrm{v}=\mathrm{v}+\varphi^{x} \frac{\partial}{\partial u_{x}}+\varphi^{y} \frac{\partial}{\partial u_{y}}+\varphi^{z} \frac{\partial}{\partial u_{z}}+\varphi^{t} \frac{\partial}{\partial u_{t}}+\psi^{x} \frac{\partial}{\partial v_{x}}+\psi^{y} \frac{\partial}{\partial v_{y}} \\
+\psi^{z} \frac{\partial}{\partial v_{z}}+\psi^{t} \frac{\partial}{\partial v_{t}}+\chi^{x} \frac{\partial}{\partial w_{x}}+\chi^{y} \frac{\partial}{\partial w_{y}}+\chi^{z} \frac{\partial}{\partial w_{z}}+\chi^{t} \frac{\partial}{\partial w_{t}}, \\
p r^{(2)} \mathrm{v}=p r^{(1)} v+\phi^{x x} \frac{\partial}{\partial u_{x x}}+\phi^{y y} \frac{\partial}{\partial u_{y y}}+\phi^{z z} \frac{\partial}{\partial u_{z z}}+\phi^{t t} \frac{\partial}{\partial u_{t t}}+\psi^{x x} \frac{\partial}{\partial v_{x x}}+\psi^{y y} \frac{\partial}{\partial v_{y y}}+\psi^{z z} \frac{\partial}{\partial v_{z z}} \\
+\psi^{t t} \frac{\partial}{\partial v_{t t}}+\chi^{x x} \frac{\partial}{\partial w_{x x}}+\chi^{y y} \frac{\partial}{\partial w_{y y}}+\chi^{z z} \frac{\partial}{\partial w_{z z}}+\chi^{t t} \frac{\partial}{\partial w_{t t}}+\phi^{x t} \frac{\partial}{\partial u_{x t}}+\phi^{y t} \frac{\partial}{\partial u_{y t}} \\
+\phi^{z t} \frac{\partial}{\partial u_{z t}}+\psi^{x t} \frac{\partial}{\partial v_{x t}}+\psi^{y t} \frac{\partial}{\partial v_{y t}}+\psi^{z t} \frac{\partial}{\partial v_{z t}}+\chi^{x t} \frac{\partial}{\partial w_{x t}}+\chi^{y t} \frac{\partial}{\partial w_{y t}}+\chi^{z t} \frac{\partial}{\partial w_{z t}} .
\end{gathered}
$$

Applying $p r^{(2)} \mathrm{V}$ to (4), we find the following system of symmetry equations 


$$
\left\{\begin{array}{l}
\phi^{t}=v\left(\chi^{x x}+\chi^{y y}+\chi^{z z}\right)-w\left(\psi^{x x}+\psi^{y y}+\psi^{z z}\right)+\left(w_{x x}+w_{y y}+w_{z z}\right) \psi-\left(v_{x x}+v_{y y}+v_{z z}\right) \chi, \\
\psi^{t}=w\left(\phi^{x x}+\phi^{y y}+\phi^{z z}\right)-u\left(\chi^{x x}+\chi^{y y}+\chi^{z z}\right)+\left(u_{x x}+u_{y y}+u_{z z}\right) \chi-\left(w_{x x}+w_{y y}+w_{z z}\right) \phi, \\
\chi^{t}=u\left(\psi^{x x}+\psi^{y y}+\psi^{z z}\right)-v\left(\phi^{x x}+\phi^{y y}+\phi^{z z}\right)+\left(v_{x x}+v_{y y}+v_{z z}\right) \phi-\left(u_{x x}+u_{y y}+u_{z z}\right) \psi,
\end{array}\right.
$$

which must be satisfied whenever u satisfy (1). Here $\phi^{t}, \psi^{x}$, etc. are the coefficients of the first order derivatives $\frac{\partial}{\partial u_{x}}, \frac{\partial}{\partial v_{x}}$, etc. appearing in $p r^{(2)} \mathrm{v}$.

According to the formula $\phi_{\alpha}^{J}\left(x, u^{(n)}\right)=D_{J}\left(\phi_{\alpha}-\sum \xi^{i} u_{i}^{\alpha}\right)+\sum \xi^{i} u_{J, i}^{\alpha}$, we have

$$
\phi^{x x}=D_{x}^{2} \phi-u_{x} D_{x}^{2} \xi-u_{y} D_{x}^{2} \eta-u_{z} D_{x}^{2} \varsigma-u_{t} D_{x}^{2} \tau-2 u_{x x} D_{x} \xi-2 u_{x y} D_{x} \eta-2 u_{x z} D_{x} \varsigma-2 u_{x t} D_{x} \tau .
$$

Similarly, we can get $\phi^{t}, \psi^{t}, \chi^{t}, \phi^{y y}, \phi^{z z}, \psi^{x x}, \psi^{y y}, \psi^{z z}, \chi^{x x}, \chi^{y y}, \chi^{z z}$.

we find the determining equations for the symmetry group of the (1) Equation (5) to be the following:

$$
\left\{\begin{array}{l}
v\left(\chi_{x x}+\chi_{y y}+\chi_{z z}\right)-w\left(\psi_{x x}+\psi_{y y}+\psi_{z z}\right)-\phi_{t}=0, \\
w\left(\tau_{t}-2 \xi_{x}\right)+\chi=0, v\left(\tau_{t}-2 \xi_{x}\right)+\psi=0, u\left(\tau_{t}-2 \xi_{x}\right)+\phi=0, \\
v\left(2 \chi_{w x}-\xi_{x x}-\xi_{y y}-\xi_{z z}\right)=0, v\left(2 \chi_{w y}-\eta_{x x}-\eta_{y y}-\eta_{z z}\right)=0, v\left(2 \chi_{w z}-\varsigma_{x x}-\varsigma_{y y}-\varsigma_{z z}\right)=0, \\
\eta_{x}=-\xi_{y}, \varsigma_{x}=-\xi_{z}, \varsigma_{y}=-\eta_{z} .
\end{array}\right.
$$

Since we have now satisfied all the determining equations, we conclude that most general infinitesimal symmetry of (1) has coefficient functions of the form:

$$
\begin{gathered}
\xi=c_{1} x+c_{2} y+c_{3} z+c_{5}, \\
\eta=-c_{2} x+c_{1} y+c_{4} z+c_{6}, \\
\varsigma=-c_{3} x-c_{4} y+c_{1} z+c_{7}, \\
\tau=c_{1} t+c_{8}, \\
\phi=c_{1} u, \\
\psi=c_{1} v, \\
\chi=c_{1} w,
\end{gathered}
$$

where $c_{1}, \cdots, c_{8}$ are arbitrary constants. Thus the Lie-algebra of infinitesimal of the Landau-Lifshitz equation is spanned by eight vector fields:

$$
\begin{aligned}
\mathrm{v}_{1}=x \partial_{x}+y \partial_{y}+z \partial_{z}+t \partial_{t}+u \partial_{u}+v \partial_{v}+w \partial_{w}, \\
\mathrm{v}_{2}=y \partial_{x}-x \partial_{y}, \\
\mathrm{v}_{3}=z \partial_{x}-x \partial_{z}, \\
\mathrm{v}_{4}=z \partial_{x}-y \partial_{z}, \\
\mathrm{v}_{5}=\partial_{x}, \\
\mathrm{v}_{6}=\partial_{y}, \\
\mathrm{v}_{7}=\partial_{z}, \\
\mathrm{v}_{8}=\partial_{t},
\end{aligned}
$$

so we have

$$
\mathrm{v}=c_{1} \mathrm{v}_{1}+c_{2} \mathrm{v}_{2}+\cdots+c_{8} \mathrm{v}_{8} .
$$

The one-parameter groups $G_{i}$ generated by the $\mathrm{v}_{i}$. The entries give the transformed point 
$\exp \left(\varepsilon \mathrm{v}_{i}\right)(x, y, z, t, u, v, w)=(\bar{x}, \bar{y}, \bar{z}, \bar{t}, \bar{u}, \bar{v}, \bar{w}):$

$$
\begin{gathered}
G_{1}:\left(\mathrm{e}^{\varepsilon} x, \mathrm{e}^{\varepsilon} y, \mathrm{e}^{\varepsilon} z, \mathrm{e}^{\varepsilon} t, \mathrm{e}^{\varepsilon} u, \mathrm{e}^{\varepsilon} v, \mathrm{e}^{\varepsilon} w\right), \\
G_{2}:(x+\varepsilon y, y-\varepsilon x, z, t, u, v, w), \\
G_{3}:(x+\varepsilon z, y, z-\varepsilon x, t, u, v, w), \\
G_{4}:(x, y+\varepsilon z, z-\varepsilon y, t, u, v, w), \\
G_{5}:(x+\varepsilon, y, z, t, u, v, w), \\
G_{6}:(x, y+\varepsilon, z, t, u, v, w), \\
G_{7}:(x, y, z+\varepsilon, t, u, v, w), \\
G_{8}:(x, y, z, t+\varepsilon, u, v, w),
\end{gathered}
$$

where $G_{1}$ is a Galiean transformation, $G_{2}, G_{3}, G_{4}, G_{5}, G_{6}, G_{7}$ are space translations, $G_{8}$ is a time translation. $\varepsilon$ is an arbitrary constant.

Theorem 1. If $u=f(x, y, z, t), v=g(x, y, z, t), w=h(x, y, z, t)$ are known solutions of (1), then by using the symmetry groups $G_{i}(i=1,2, \cdots, 8)$, so are the functions

$$
\begin{gathered}
u_{1}=\mathrm{e}^{\varepsilon} f\left(\mathrm{e}^{-\varepsilon} x, \mathrm{e}^{-\varepsilon} y, \mathrm{e}^{-\varepsilon} z, \mathrm{e}^{-\varepsilon} t\right), \\
v_{1}=\mathrm{e}^{\varepsilon} g\left(\mathrm{e}^{-\varepsilon} x, \mathrm{e}^{-\varepsilon} y, \mathrm{e}^{-\varepsilon} z, \mathrm{e}^{-\varepsilon} t\right), \\
w_{1}=\mathrm{e}^{\varepsilon} h\left(\mathrm{e}^{-\varepsilon} x, \mathrm{e}^{-\varepsilon} y, \mathrm{e}^{-\varepsilon} z, \mathrm{e}^{-\varepsilon} t\right), \\
u_{2}=f\left(\frac{x-\varepsilon y}{1+\varepsilon^{2}}, \frac{\varepsilon x+y}{1+\varepsilon^{2}}, z, t\right), v_{2}=g\left(\frac{x-\varepsilon y}{1+\varepsilon^{2}}, \frac{\varepsilon x+y}{1+\varepsilon^{2}}, z, t\right), w_{2}=h\left(\frac{x-\varepsilon y}{1+\varepsilon^{2}}, \frac{\varepsilon x+y}{1+\varepsilon^{2}}, z, t\right), \\
u_{3}=f\left(\frac{x-\varepsilon z}{1+\varepsilon^{2}}, y, \frac{\varepsilon x+z}{1+\varepsilon^{2}}, t\right), v_{3}=g\left(\frac{x-\varepsilon z}{1+\varepsilon^{2}}, y, \frac{\varepsilon x+z}{1+\varepsilon^{2}}, t\right), w_{3}=h\left(\frac{x-\varepsilon z}{1+\varepsilon^{2}}, y, \frac{\varepsilon x+z}{1+\varepsilon^{2}}, t\right), \\
u_{4}=f\left(x, \frac{y-\varepsilon z}{1+\varepsilon^{2}}, \frac{\varepsilon y+z}{1+\varepsilon^{2}}, t\right), v_{4}=g\left(x, \frac{y-\varepsilon z}{1+\varepsilon^{2}}, \frac{\varepsilon y+z}{1+\varepsilon^{2}}, t\right), w_{4}=h\left(x, \frac{y-\varepsilon z}{1+\varepsilon^{2}}, \frac{\varepsilon y+z}{1+\varepsilon^{2}}, t\right), \\
u_{5}=f(x-\varepsilon, y, z, t), v_{5}=g(x-\varepsilon, y, z, t), w_{5}=h(x-\varepsilon, y, z, t) \\
u_{6}=f(x, y-\varepsilon, z, t), v_{6}=f(x, y-\varepsilon, z, t), w_{6}=f(x, y-\varepsilon, z, t) \\
u_{7}=f(x, y, z-\varepsilon, t), v_{7}=f(x, y, z-\varepsilon, t), w_{7}=f(x, y, z-\varepsilon, t) \\
u_{8}=f(x, y, z, t-\varepsilon), v_{8}=f(x, y, z, t-\varepsilon), w_{8}=f(x, y, z, t-\varepsilon),
\end{gathered}
$$

where $\varepsilon$ is any real number.

For the known solutions $u=f(x, y, z, t), v=g(x, y, z, t), w=h(x, y, z, t)$, by using one-parameter symmetry groups $G_{i}(i=1,2, \cdots, 8)$ continuously, we can obtain a new solution which can be expressed as the following form:

$$
\begin{aligned}
u= & \mathrm{e}^{\varepsilon_{1}} f\left(\mathrm{e}^{-\varepsilon_{1}}\left(\frac{1}{1+\varepsilon_{2}^{2}}+\frac{1}{1+\varepsilon_{3}^{2}}\right) x-\frac{\varepsilon_{2}}{1+\varepsilon_{2}^{2}} y-\frac{\varepsilon_{3}}{1+\varepsilon_{3}^{2}} z-\varepsilon_{5},\right. \\
& \mathrm{e}^{-\varepsilon_{1}}\left(\frac{1}{1+\varepsilon_{2}^{2}}+\frac{1}{1+\varepsilon_{4}^{2}}\right) y+\frac{\varepsilon_{2}}{1+\varepsilon_{2}^{2}} x-\frac{\varepsilon_{4}}{1+\varepsilon_{4}^{2}} z-\varepsilon_{6}, \\
& \left.\mathrm{e}^{-\varepsilon_{1}}\left(\frac{1}{1+\varepsilon_{3}^{2}}+\frac{1}{1+\varepsilon_{4}^{2}}\right) z+\frac{\varepsilon_{3}}{1+\varepsilon_{3}^{2}} x+\frac{\varepsilon_{4}}{1+\varepsilon_{4}^{2}} y-\varepsilon_{7}, e^{-\varepsilon_{1}} t-\varepsilon_{8}\right),
\end{aligned}
$$




$$
\begin{aligned}
v= & \mathrm{e}^{\varepsilon_{1}} g\left(\mathrm{e}^{-\varepsilon_{1}}\left(\frac{1}{1+\varepsilon_{2}^{2}}+\frac{1}{1+\varepsilon_{3}^{2}}\right) x-\frac{\varepsilon_{2}}{1+\varepsilon_{2}^{2}} y-\frac{\varepsilon_{3}}{1+\varepsilon_{3}^{2}} z-\varepsilon_{5},\right. \\
& \mathrm{e}^{-\varepsilon_{1}}\left(\frac{1}{1+\varepsilon_{2}^{2}}+\frac{1}{1+\varepsilon_{4}^{2}}\right) y+\frac{\varepsilon_{2}}{1+\varepsilon_{2}^{2}} x-\frac{\varepsilon_{4}}{1+\varepsilon_{4}^{2}} z-\varepsilon_{6}, \\
& \left.\mathrm{e}^{-\varepsilon_{1}}\left(\frac{1}{1+\varepsilon_{3}^{2}}+\frac{1}{1+\varepsilon_{4}^{2}}\right) z+\frac{\varepsilon_{3}}{1+\varepsilon_{3}^{2}} x+\frac{\varepsilon_{4}}{1+\varepsilon_{4}^{2}} y-\varepsilon_{7}, e^{-\varepsilon_{1}} t-\varepsilon_{8}\right), \\
w= & \mathrm{e}^{\varepsilon_{1}} h\left(\mathrm{e}^{-\varepsilon_{1}}\left(\frac{1}{1+\varepsilon_{2}^{2}}+\frac{1}{1+\varepsilon_{3}^{2}}\right) x-\frac{\varepsilon_{2}}{1+\varepsilon_{2}^{2}} y-\frac{\varepsilon_{3}}{1+\varepsilon_{3}^{2}} z-\varepsilon_{5},\right. \\
& \mathrm{e}^{-\varepsilon_{1}}\left(\frac{1}{1+\varepsilon_{2}^{2}}+\frac{1}{1+\varepsilon_{4}^{2}}\right) y+\frac{\varepsilon_{2}}{1+\varepsilon_{2}^{2}} x-\frac{\varepsilon_{4}}{1+\varepsilon_{4}^{2}} z-\varepsilon_{6}, \\
& \left.\mathrm{e}^{-\varepsilon_{1}}\left(\frac{1}{1+\varepsilon_{3}^{2}}+\frac{1}{1+\varepsilon_{4}^{2}}\right) z+\frac{\varepsilon_{3}}{1+\varepsilon_{3}^{2}} x+\frac{\varepsilon_{4}}{1+\varepsilon_{4}^{2}} y-\varepsilon_{7}, e^{-\varepsilon_{1}} t-\varepsilon_{8}\right),
\end{aligned}
$$

where $\varepsilon_{i}(i=1,2, \cdots, 8)$ are arbitrary constants.

In vector notation, the system has the form

$$
\mathrm{u}_{t}=-\mathrm{u} \times \Delta \mathrm{u}-\lambda \mathrm{u} \times(\mathrm{u} \times \Delta \mathrm{u}),
$$

when $\lambda_{1}=-1, \lambda_{2}=-\lambda(\lambda>0)$ in (2).

In view of the vector identities

$$
u \times(u \times \Delta u)=(u \cdot \Delta u) u-|u|^{2} \Delta u,
$$

Equation (6) can equivalently be written as

$$
\mathrm{u}_{t}=-\mathrm{u} \times \Delta \mathrm{u}-\lambda\left[(\mathrm{u} \cdot \Delta, \mathrm{u}) \mathrm{u}-|\mathrm{u}|^{2} \Delta \mathrm{u}\right] .
$$

We transformed equations as follows:

$$
\left\{\begin{array}{l}
u_{t}=w \Delta v-v \Delta w-\lambda\left(u v \Delta v+u w \Delta w-v^{2} \Delta u-w^{2} \Delta u\right), \\
v_{t}=u \Delta w-w \Delta u-\lambda\left(u v \Delta u+v w \Delta w-u^{2} \Delta v-w^{2} \Delta v\right), \\
w_{t}=v \Delta u-u \Delta v-\lambda\left(u w \Delta u+v w \Delta v-u^{2} \Delta w-v^{2} \Delta w\right) .
\end{array}\right.
$$

Applying $p r^{(2)} \mathrm{v}$ to (8), we find the following system of symmetry equations

$$
\left\{\begin{aligned}
\phi^{t}= & w\left(\psi^{x x}+\psi^{y y}+\psi^{z z}\right)+\chi \Delta v-v\left(\chi^{x x}+\chi^{y y}+\chi^{z z}\right)-\psi \Delta w \\
& -\lambda\left[\phi v \Delta v+\psi u \Delta v+u v\left(\psi^{x x}+\psi^{y y}+\psi^{z z}\right)+\phi w \Delta w+\chi u \Delta w+u w\left(\chi^{x x}+\chi^{y y}+\chi^{z z}\right)\right. \\
& \left.-2 \psi v \Delta u-v^{2}\left(\phi^{x x}+\phi^{y y}+\phi^{z z}\right)-2 \chi w \Delta u-w^{2}\left(\phi^{x x}+\phi^{y y}+\phi^{z z}\right)\right], \\
\psi^{t}= & u\left(\chi^{x x}+\chi^{y y}+\chi^{z z}\right)+\phi \Delta w-w\left(\phi^{x x}+\phi^{y y}+\phi^{z z}\right)-\chi \Delta u \\
& -\lambda\left[\phi v \Delta u+\psi u \Delta u+u v\left(\phi^{x x}+\phi^{y y}+\phi^{z z}\right)+\chi v \Delta w+\psi w \Delta w+w v\left(\chi^{x x}+\chi^{y y}+\chi^{z z}\right)\right. \\
& \left.-2 \phi u \Delta v-u^{2}\left(\psi^{x x}+\psi^{y y}+\psi^{z z}\right)-2 \chi w \Delta v-w^{2}\left(\psi^{x x}+\psi^{y y}+\psi^{z z}\right)\right] \\
\chi^{t}= & v\left(\phi^{x x}+\phi^{y y}+\phi^{z z}\right)+\psi \Delta u-u\left(\psi^{x x}+\psi^{y y}+\psi^{z z}\right)-\phi \Delta v \\
& -\lambda\left[\phi w \Delta u+\chi u \Delta u+u w\left(\phi^{x x}+\phi^{y y}+\phi^{z z}\right)+\psi w \Delta v+\chi v \Delta+v w\left(\psi^{x x}+\psi^{y y}+\psi^{z z}\right)\right. \\
& \left.-2 \phi u \Delta w-u^{2}\left(\chi^{x x}+\chi^{y y}+\chi^{z z}\right)-2 \psi v \Delta w-v^{2}\left(\chi^{x x}+\chi^{y y}+\chi^{z z}\right)\right] .
\end{aligned}\right.
$$


Then use the same method, we can find most generated infinitesimal symmetry of (6) has coefficient functions of the form:

$$
\begin{aligned}
& \xi=c_{1}, \\
& \eta=c_{2}, \\
& \varsigma=c_{3}, \\
& \tau=c_{4}, \\
& \phi=c_{5}, \\
& \psi=c_{6}, \\
& \chi=c_{7},
\end{aligned}
$$

where $c_{1}, \cdots, c_{7}$ are arbitrary constants. Thus the Lie-algebra of infinitesimal of the Landau-Lifshitz-Gilbert equation equation is spanned by seven vector fields:

$$
\begin{aligned}
\mathrm{v}_{1} & =\partial_{x}, \\
\mathrm{v}_{2} & =\partial_{y}, \\
\mathrm{v}_{3} & =\partial_{z}, \\
\mathrm{v}_{4} & =\partial_{t}, \\
\mathrm{v}_{5} & =\partial_{u}, \\
\mathrm{v}_{6} & =\partial_{v}, \\
\mathrm{v}_{7} & =\partial_{w} .
\end{aligned}
$$

So we have

$$
\mathrm{v}=c_{1} \mathrm{v}_{1}+c_{2} \mathrm{v}_{2}+c_{3} \mathrm{v}_{3}+c_{4} \mathrm{v}_{4}+c_{5} \mathrm{v}_{5}+c_{6} \mathrm{v}_{6}+c_{7} \mathrm{v}_{7},
$$

where $G_{1}, G_{2}, G_{3}$ are space transformations, $G_{4}$ is a space translation, $G_{5}, G_{6}, G_{7}$ are Galiean translations, $\varepsilon$ is an arbitrary constant.

Theorem 2. If $u=f(x, y, z, t), v=g(x, y, z, t), w=h(x, y, z, t)$ are known solutions of (6), then by using the symmetry groups $G_{i}(i=1,2, \cdots, 7)$, so are the functions

$$
\begin{gathered}
u_{1}=f(x-\varepsilon, y, z, t), v_{1}=g(x-\varepsilon, y, z, t), w_{1}=h(x-\varepsilon, y, z, t), \\
u_{2}=f(x, y-\varepsilon, z, t), v_{2}=g(x, y-\varepsilon, z, t), w_{2}=h(x, y-\varepsilon, z, t), \\
u_{3}=f(x, y, z-\varepsilon, t), v_{3}=g(x, y, z-\varepsilon, t), w_{3}=h(x, y, z-\varepsilon, t), \\
u_{4}=f(x, y, z, t-\varepsilon), v_{4}=g(x, y, z, t-\varepsilon), w_{4}=h(x, y, z, t-\varepsilon), \\
u_{5}=f(x, y, z, t)+\varepsilon, v_{5}=g(x, y, z, t), w_{5}=h(x, y, z, t), \\
u_{6}=f(x, y, z, t), v_{6}=g(x, y, z, t)+\varepsilon, w_{6}=h(x, y, z, t), \\
u_{7}=f(x, y, z, t), v_{7}=g(x, y, z, t), w_{7}=h(x, y, z, t)+\varepsilon,
\end{gathered}
$$

where $\varepsilon$ is any real number.

For the known solutions $u=f(x, y, z, t), v=g(x, y, z, t), w=h(x, y, z, t)$, by using one-parameter symmetry groups $G_{i}(i=1,2, \cdots, 7)$ continuously, we can obtain a new solution which can be expressed as the following form:

$$
\begin{aligned}
& u=f\left(x-\varepsilon_{1}, y-\varepsilon_{2}, z-\varepsilon_{3}, t-\varepsilon_{4}\right)+\varepsilon_{5}, \\
& v=g\left(x-\varepsilon_{1}, y-\varepsilon_{2}, z-\varepsilon_{3}, t-\varepsilon_{4}\right)+\varepsilon_{6},
\end{aligned}
$$




$$
w=h\left(x-\varepsilon_{1}, y-\varepsilon_{2}, z-\varepsilon_{3}, t-\varepsilon_{4}\right)+\varepsilon_{7},
$$

where $\varepsilon_{i}(i=1,2, \cdots, 7)$ are arbitrary constants.

Remark If $\mathrm{u}=(u, v, w)$ is a known solution, we can get $\mathrm{v}=A \mathrm{u}$ is a new solution through Lie group method, where $A$ is arbitrary constant orthogonal matrices.

\section{Exact Solutions of the Landau-Lifshitz Equation}

In this section, we choose the known blow-up solutions and explicit dynamic spherical cone symmetric solutions from [10] and [14] to get the relevant group invariant solutions.

According to [10],

$$
\left\{\begin{array}{l}
\mathrm{u}_{t}=\mathrm{u} \times \Delta \mathrm{u}, \mathrm{u} \in \mathbb{R}^{3}, t \in \mathbb{R}^{+}, \\
\mathrm{u}(\mathrm{x}, 0)=\left(k_{1}(x+y+z), k_{2} \cos \frac{x+y+z}{3 k_{1} T},-k_{2} \sin \frac{x+y+z}{3 k_{1} T}\right) \in C^{\infty}\left(\mathbb{R}^{3}\right),
\end{array}\right.
$$

has a blow-up solution:

$$
\left\{\begin{array}{l}
u(x, y, z, t)=k_{1}(x+y+z), \\
v(x, y, z, t)=k_{2} \cos \frac{x+y+z}{3 k_{1}(T-t)}, \\
w(x, y, z, t)=-k_{2} \sin \frac{x+y+z}{3 k_{1}(T-t)},
\end{array}\right.
$$

where $u \in C^{\infty}\left(\mathbb{R}^{3} \times[0, T)\right)$.

Case 1 Using $u_{1}$ in Theorem 1, we get the new blow-up solutions of (1) as follows:

$$
\left\{\begin{array}{l}
u(x, y, z, t)=k_{1} \mathrm{e}^{\varepsilon}(x+y+z), \\
v(x, y, z, t)=k_{2} \mathrm{e}^{\varepsilon} \cos \frac{\mathrm{e}^{-\varepsilon}(x+y+z)}{3 k_{1}(T-t)}, \\
w(x, y, z, t)=-k_{2} \mathrm{e}^{\varepsilon} \sin \frac{\mathrm{e}^{-\varepsilon}(x+y+z)}{3 k_{1}(T-t)},
\end{array}\right.
$$

where $u \in C^{\infty}\left(\mathbb{R}^{3} \times[0, T)\right)$ satisfying the following initial value:

$$
\left\{\begin{array}{l}
u(x, y, z, 0)=k_{1} \mathrm{e}^{\varepsilon}(x+y+z), \\
v(x, y, z, 0)=k_{2} \mathrm{e}^{\varepsilon} \cos \frac{\mathrm{e}^{-\varepsilon}(x+y+z)}{3 k_{1} T}, \\
w(x, y, z, 0)=-k_{2} \mathrm{e}^{\varepsilon} \sin \frac{\mathrm{e}^{-\varepsilon}(x+y+z)}{3 k_{1} T} .
\end{array}\right.
$$

Case 2 Using $u_{2}$ in Theorem 1, we get the new blow-up solutions of (1) as follows:

$$
\left\{\begin{array}{l}
u(x, y, z, t)=3 k_{1} \frac{1+\varepsilon^{2}}{\varepsilon^{2}+3}\left(\frac{1+\varepsilon}{1+\varepsilon^{2}} x+\frac{1-\varepsilon}{1+\varepsilon^{2}} y+z\right) \\
v(x, y, z, t)=k_{2} \cos \frac{\frac{1+\varepsilon}{1+\varepsilon^{2}} x+\frac{1-\varepsilon}{1+\varepsilon^{2}} y+z}{3 k_{1}(T-t)} \\
w(x, y, z, t)=-k_{2} \sin \frac{\frac{1+\varepsilon}{1+\varepsilon^{2}} x+\frac{1-\varepsilon}{1+\varepsilon^{2}} y+z}{3 k_{1}(T-t)}
\end{array}\right.
$$

where $u \in C^{\infty}\left(\mathbb{R}^{3} \times[0, T)\right)$ satisfying the following initial value: 


$$
\left\{\begin{array}{l}
u(x, y, z, 0)=3 k_{1} \frac{1+\varepsilon^{2}}{\varepsilon^{2}+3}\left(\frac{1+\varepsilon}{1+\varepsilon^{2}} x+\frac{1-\varepsilon}{1+\varepsilon^{2}} y+z\right) \\
v(x, y, z, 0)=k_{2} \cos \frac{\frac{1+\varepsilon}{1+\varepsilon^{2}} x+\frac{1-\varepsilon}{1+\varepsilon^{2}} y+z}{3 k_{1} T} \\
w(x, y, z, 0)=-k_{2} \sin \frac{\frac{1+\varepsilon}{1+\varepsilon^{2}} x+\frac{1-\varepsilon}{1+\varepsilon^{2}} y+z}{3 k_{1} T}
\end{array}\right.
$$

According to [10],

$$
\left\{\begin{array}{l}
\mathrm{u}_{t}=\mathrm{u} \times \mathrm{u}_{r r}+\frac{n-1}{r} \mathrm{u} \times \mathrm{u}_{r}, \\
\mathrm{u}(x, 0)=r^{-n}\left(k_{1}, k_{2} \cos \frac{r^{n+2}}{k_{1}(n+2)^{2} T},-k_{2} \sin \frac{r^{n+2}}{k_{1}(n+2)^{2} T}\right), \\
\mathrm{u} \times\left.\mathrm{u}\right|_{r=1}=k_{1}^{2}+k_{2}^{2}, \mathrm{u} \times\left.\mathrm{u}\right|_{r=2}=2^{-2 n}\left(k_{1}^{2}+k_{2}^{2}\right),
\end{array}\right.
$$

for $r=\sqrt{x^{2}+y^{2}+z^{2}}$, has a blow-up solution:

$$
\left\{\begin{array}{l}
\mathrm{u}(r, t)=r^{-n}\left(k_{1}, k_{2} \cos \theta,-k_{2} \sin \theta\right), \\
\theta=-\frac{r^{n+2}}{k_{1}(n+2)^{2}(T-t)}
\end{array}\right.
$$

Case 3 Using $u_{1}$ in Theorem 1, we get the new blow-up solutions for the cylindrical symmetric LandauLifshitz Equation (3), typically $n=3$ as follows:

$$
\left\{\begin{array}{l}
\mathrm{u}(r, t)=\mathrm{e}^{4 \varepsilon}\left(k_{1} r^{-3}, k_{2} r^{-3} \cos \theta,-k_{2} r^{-3} \sin \theta\right) \\
\theta=-\frac{\mathrm{e}^{-5 \varepsilon} r^{5}}{25 k_{1}\left(T-\mathrm{e}^{-\varepsilon} t\right)}
\end{array}\right.
$$

for $r=\sqrt{x^{2}+y^{2}+z^{2}}$, satisfying the following initial value:

$$
\left\{\begin{array}{l}
\mathrm{u}(\mathrm{x}, 0)=\mathrm{e}^{4 \varepsilon} r^{-3}\left(k_{1}, k_{2} \cos \frac{\mathrm{e}^{-5 \varepsilon} r^{5}}{25 k_{1} T},-k_{2} \sin \frac{\mathrm{e}^{-5 \varepsilon} r^{5}}{25 k_{1} T}\right), \\
\mathrm{u} \times\left.\mathrm{u}\right|_{r=1}=\mathrm{e}^{4 \varepsilon}\left(k_{1}^{2}+k_{2}^{2}\right), \mathrm{u} \times\left.\mathrm{u}\right|_{r=2}=\mathrm{e}^{4 \varepsilon} 2^{-6}\left(k_{1}^{2}+k_{2}^{2}\right) .
\end{array}\right.
$$

According to [14],

$$
\left\{\begin{array}{l}
\mathrm{u}_{t}=\mathrm{u} \times \Delta \mathrm{u}, \Omega \times(0, T), \\
\mathrm{u}(\mathrm{x}, 0)=\left(\cos \theta_{0}, \sin \theta_{0}, 0\right), \theta_{0}=\frac{c_{1}\left[(x-y)^{2}+(x-z)^{2}+(z-y)^{2}\right]}{12 \sqrt{1+c_{2}^{2}}},
\end{array}\right.
$$

has the explicit dynamic spherical cone symmetric solutions:

$$
\left\{\begin{array}{l}
\mathrm{u}=\frac{1}{\sqrt{1+\left(c_{1} t+c_{2}\right)^{2}}}\left(\cos \theta, \sin \theta, c_{1} t+c_{2}\right), \\
\theta=\frac{c_{1}\left[(x-y)^{2}+(x-z)^{2}+(z-y)^{2}\right]}{12 \sqrt{1+\left(c_{1} t+c_{2}\right)^{2}}} .
\end{array}\right.
$$

Case 4 Using $u_{1}$ in Theorem 1, we get the new explicit dynamic spherical cone symmetric solutions of (1) 
as follows:

$$
\left\{\begin{array}{l}
\mathrm{u}=\frac{1}{\sqrt{1+\left(c_{1} \mathrm{e}^{-\varepsilon} t+c_{2}\right)^{2}}}\left(\cos \theta, \sin \theta, c_{1} \mathrm{e}^{-\varepsilon} t+c_{2}\right), \\
\theta=\frac{c_{1} \mathrm{e}^{-2 \varepsilon}\left[(x-y)^{2}+(x-z)^{2}+(z-y)^{2}\right]}{12 \sqrt{1+\left(c_{1} \mathrm{e}^{-\varepsilon} t+c_{2}\right)^{2}}},
\end{array}\right.
$$

satisfying the following initial value:

$$
\left\{\begin{array}{l}
\mathrm{u}(\mathrm{x}, 0)=\left(\cos \theta_{0}, \sin \theta_{0}, 0\right), \\
\theta_{0}=\frac{c_{1} \mathrm{e}^{-2 \varepsilon}\left[(x-y)^{2}+(x-z)^{2}+(z-y)^{2}\right]}{12 \sqrt{1+c_{2}^{2}}} .
\end{array}\right.
$$

Case 5 Using $u_{2}$ in Theorem 1, we get the new explicit dynamic spherical cone symmetric solutions of (1) as follows:

$$
\left\{\begin{array}{l}
u=\frac{1}{\sqrt{1+\left(c_{1} t+c_{2}\right)^{2}}}\left(\cos \theta, \sin \theta, c_{1} t+c_{2}\right), \\
\theta=\frac{c_{1}\left[\varepsilon^{2}(x+y)^{2}+(x-y)^{2}+(x-z)^{2}+(z-y)^{2}+\varepsilon x^{2}(\varepsilon-2)+\varepsilon y^{2}(\varepsilon+2)+2 \varepsilon x y z\right]}{12\left(1+\varepsilon^{2}\right)^{2} \sqrt{1+\left(c_{1} t+c_{2}\right)^{2}}},
\end{array}\right.
$$

satisfying the following initial value:

$$
\left\{\begin{array}{l}
\mathrm{u}(\mathrm{x}, 0)=\mathrm{e}^{\varepsilon}\left(\cos \theta_{0}, \sin \theta_{0}, 0\right), \\
\theta_{0}=\frac{c_{1} \mathrm{e}^{-2 \varepsilon}\left[(x-y)^{2}+(x-z)^{2}+(z-y)^{2}\right]}{12 \sqrt{1+c_{2}^{2}}} .
\end{array}\right.
$$

Remark Similarly, we can utilize the different seed solutions of [10] [14], repeatedly using $\mathrm{u}_{i}(i=1,2, \cdots, 8)$ to obtain different group invariant solutions, so extend the known exact solutions in [9] [13].

\section{Uniqueness and Stability of the Landau-Lifshitz Equation}

In this section, we study the uniqueness and stability of the initial boundary value problem for (1) and we have the following results and it should be results that matter instead.

\subsection{Uniqueness}

Theorem 3. There exists $\Omega \in \mathbb{R}^{3}$ is a bounded domain and $k_{j}(j=1,2)$ are nonzero constants. Then we the following initial value problem:

$$
\left\{\begin{array}{l}
\boldsymbol{u}_{t}=\boldsymbol{u} \times \Delta \boldsymbol{u}, u \in \mathbb{R}^{3}, t \in \mathbb{R}^{+}, \\
\boldsymbol{u}(\boldsymbol{x}, 0)=\left(k_{1} \mathrm{e}^{\varepsilon}(x+y+z), k_{2} \mathrm{e}^{\varepsilon} \cos \frac{\mathrm{e}^{-\varepsilon}(x+y+z)}{3 k_{1} T},-k_{2} \mathrm{e}^{\varepsilon} \sin \frac{\mathrm{e}^{-\varepsilon}(x+y+z)}{3 k_{1} T}\right), \\
\boldsymbol{u}(\boldsymbol{x}, t)=\left(k_{1} \mathrm{e}^{\varepsilon}(x+y+z), k_{2} \mathrm{e}^{\varepsilon} \cos \frac{\mathrm{e}^{-\varepsilon}(x+y+z)}{3 k_{1}(T-t)},-k_{2} \mathrm{e}^{\varepsilon} \sin \frac{\mathrm{e}^{-\varepsilon}(x+y+z)}{3 k_{1}(T-t)}\right), \\
\text { on } \partial \Omega \times[0, T),
\end{array}\right.
$$

has a unique smooth blow-up solution $\boldsymbol{u} \in C^{\infty}\left(\mathbb{R}^{3} \times[0, T)\right)$.

Proof. To prove the uniqueness we consider two smooth solutions $\mathbf{u}, \overline{\mathbf{u}} \in C^{\infty}\left(\mathbb{R}^{3} \times 0 \leq t<T\right)$. Let their 
difference be $\rho=\mathbf{u}-\overline{\mathbf{u}}$, where $\rho=\left(\rho_{1}, \rho_{2}, \rho_{3}\right), \mathbf{u}=(u, v, w), \overline{\mathbf{u}}=(\bar{u}, \bar{v}, \bar{w})$. Then, subtracting the equations each other in (1), we have

$$
\left\{\begin{array}{l}
\rho_{t}=\rho \times \Delta \mathbf{u}+\overline{\mathbf{u}} \times \Delta \rho, \text { in } \Omega \times(0, T), \\
\rho(\mathbf{x}, 0)=(\mathbf{0}, 0), \text { in } \Omega, \\
\rho \mid \partial \Omega=(\mathbf{0}, 0) .
\end{array}\right.
$$

Multiplying the first equation of (27) by $-\Delta \rho$, integrating over $\Omega$, and using the Gauss formula [23], we obtain

$$
\begin{gathered}
\left(\rho_{t},-\Delta \rho\right)=\frac{1}{2} \frac{\mathrm{d}}{\mathrm{d} t}\|\nabla \rho\|_{L^{2}}^{2}, \\
\left(\rho_{t},-\Delta \rho\right)=(\rho \times \Delta \mathbf{u}+\overline{\mathbf{u}} \times \rho,-\Delta \rho)=\int_{\Omega} \nabla \rho \cdot(\rho \times \Delta \nabla \mathbf{u}) \mathrm{d} \mathbf{x},
\end{gathered}
$$

for $\nabla \mathbf{u}=(\nabla u, \nabla v, \nabla w), \Delta \mathbf{u}=(\Delta u, \Delta v, \Delta w), \nabla \rho=\left(\nabla \rho_{1}, \nabla \rho_{2}, \nabla \rho_{3}\right)$. By using $u(x, y, z, t)=k_{1} \mathrm{e}^{\varepsilon}(x+y+z)$, $v(x, y, z, t)=k_{2} \mathrm{e}^{\varepsilon} \cos \frac{\mathrm{e}^{-\varepsilon}(x+y+z)}{3 k_{1}(T-t)}, w(x, y, z, t)=-k_{2} \mathrm{e}^{\varepsilon} \sin \frac{\mathrm{e}^{-\varepsilon}(x+y+z)}{3 k_{1}(T-t)}$ in case 1, we obtain

$$
\nabla \mathbf{u}=\left(\begin{array}{ccc}
k_{1} \mathrm{e}^{\varepsilon} & k_{1} \mathrm{e}^{\varepsilon} & k_{1} \mathrm{e}^{\varepsilon} \\
-\frac{k_{2}}{3 k_{1}(T-t)} \sin \theta & -\frac{k_{2}}{3 k_{1}(T-t)} \sin \theta & -\frac{k_{2}}{3 k_{1}(T-t)} \sin \theta \\
-\frac{k_{2}}{3 k_{1}(T-t)} \cos \theta & -\frac{k_{2}}{3 k_{1}(T-t)} \cos \theta & -\frac{k_{2}}{3 k_{1}(T-t)} \cos \theta
\end{array}\right)
$$

where $\theta=\frac{\mathrm{e}^{-\varepsilon}(x+y+z)}{3 k_{1}(T-t)}$.

$$
\begin{aligned}
\|\Delta \nabla \boldsymbol{u}\|_{\infty} & =\max \left\{3 \Delta k_{1} \mathrm{e}^{\varepsilon}, \Delta\left(-\frac{k_{2}}{k_{1}(T-t)} \sin \theta\right), \Delta\left(-\frac{k_{2}}{k_{1}(T-t)} \cos \theta\right)\right\} \\
& =\sup \left|\frac{\mathrm{e}^{-2 \varepsilon} k_{2}}{3 k_{1}^{3}(T-t)^{3}}\right| \leq\left|c_{2}(t)\right| .
\end{aligned}
$$

Inserting (31) into (29), it follows that

$$
\begin{aligned}
\frac{1}{2} \frac{\mathrm{d}}{\mathrm{d} t}\|\nabla \rho\|_{L^{2}}^{2} & =(\rho \times \Delta \mathbf{u}+\overline{\mathbf{u}} \times \Delta \rho,-\Delta \rho) \\
& \leq\|\Delta \nabla \mathbf{u}\|_{L^{\infty}}\|\nabla \rho\|_{L^{2}}^{2} \leq \mid c_{2}(t)\|\nabla \rho\|_{L^{2}}^{2} .
\end{aligned}
$$

Thanks to the Gronwall inequality [23], we have the following:

$$
\|\nabla \rho\|_{L^{2}}^{2} \leq \exp \left(\int_{0}^{t} 2\left|c_{2}(\tau)\right| \mathrm{d} \tau\right)\|\nabla \rho(\mathbf{x}, 0)\|_{L^{2}}^{2}=0
$$

therefore we can prove the uniqueness of the solution in the sense of $C^{\infty}\left(\mathbb{R}^{3} \times[0, T)\right)$.

In a similar way, by using

$$
\left\{\begin{array}{l}
\mathbf{u}(r, t)=\mathrm{e}^{4 \varepsilon}\left(k_{1} r^{-3}, k_{2} r^{-3} \cos \theta,-k_{2} r^{-3} \sin \theta\right), \\
\theta=-\frac{\mathrm{e}^{-5 \varepsilon} r^{5}}{25 k_{1}\left(T-\mathrm{e}^{-\varepsilon} t\right)}
\end{array}\right.
$$

in case 3 , we obtain 


$$
\nabla \mathbf{u}=\left(\begin{array}{ccc}
-3 k_{1} \mathrm{e}^{4 \varepsilon} x r^{-5} & -3 k_{1} \mathrm{e}^{4 \varepsilon} y r^{-5} & -3 k_{1} \mathrm{e}^{4 \varepsilon} z r^{-5} \\
M_{1} & M_{1} & M_{1} \\
N_{1} & N_{1} & N_{1}
\end{array}\right),
$$

which $\theta=-\frac{\mathrm{e}^{-5 \varepsilon} r^{5}}{25 k_{1}\left(T-\mathrm{e}^{-\varepsilon} t\right)}, M_{1}=-3 \mathrm{e}^{4 \varepsilon} k_{2} r^{-5} x \cos \theta+\frac{\mathrm{e}^{4 \varepsilon} k_{2} r^{-6} x}{5 k_{1}(T-t)} \sin \theta, N_{1}=-\frac{k_{2}}{3 k_{1}(T-t)} \cos \theta$.

$$
\begin{aligned}
\|\Delta \nabla \mathbf{u}\|_{\infty}= & \sup ^{4 \varepsilon}\left|15 k_{1} r^{-7}+30 k_{1} r^{-7} x+y+z+105 k_{1} r^{-9}\right| x+y+\left.z\right|^{3} \\
& +\frac{k_{2}}{5 k_{1}(T-t)}\left(6 r^{-8}|x+y+z|+12 r^{-2}|x+y+z|+12 r^{-4}|x+y+z|^{3}\right) \mid \\
\leq & \left|c_{3}(t)\right|
\end{aligned}
$$

Inserting (34) into (29), it follows that

$$
\frac{1}{2} \frac{\mathrm{d}}{\mathrm{d} t}\|\nabla \rho\|_{L^{2}}^{2} \leq\|\Delta \nabla \mathbf{u}\|_{L^{\infty}}\|\nabla \rho\|_{L^{2}}^{2} \leq \mid c_{3}(t)\|\nabla \rho\|_{L^{2}}^{2} .
$$

Thanks to the Gronwall inequality, we have the following:

$$
\|\nabla \rho\|_{L^{2}}^{2} \leq \exp \left(\int_{0}^{t} 2\left|c_{3}(\tau)\right| \mathrm{d} \tau\right)\|\nabla \rho(\mathbf{x}, 0)\|_{L^{2}}^{2}=0,
$$

therefore we can get the uniqueness of the solution from this.

Theorem 4. There exists $\Omega \in \mathbb{R}^{3}$ is a bounded domain. Then we the following initial boundary value problem:

$$
\left\{\begin{array}{l}
\boldsymbol{u}_{t}=\boldsymbol{u} \times \Delta \boldsymbol{u}, \Omega \times(0, T), \\
\boldsymbol{u}(\boldsymbol{x}, 0)=\left(\cos \theta_{0}, \sin \theta_{0}, 0\right), \theta_{0}=\frac{c_{1} \mathrm{e}^{-2 \varepsilon}\left[(x-y)^{2}+(x-z)^{2}+(z-y)^{2}\right]}{12 \sqrt{1+c_{2}^{2}}}, \\
\boldsymbol{u}(\boldsymbol{x}, t)=\frac{1}{\sqrt{1+\left(c_{1} \mathrm{e}^{-\varepsilon} t+c_{2}\right)^{2}}}\left(\cos \theta, \sin \theta, c_{1} \mathrm{e}^{-\varepsilon} t+c_{2}\right), \text { on } \partial \Omega \times(0, T), \\
\theta=\frac{c_{1} \mathrm{e}^{-2 \varepsilon}\left[(x-y)^{2}+(x-z)^{2}+(z-y)^{2}\right]}{12 \sqrt{1+\left(c_{1} \mathrm{e}^{-\varepsilon} t+c_{2}\right)^{2}}},
\end{array}\right.
$$

has a unique explicit dynamic spherical cone symmetric solution

$$
\mathbf{u} \in C^{1}\left([0, T) ; C^{2}\left(\mathbb{R}^{3}\right)\right) \cap C^{1}\left([0, T) ; H^{2}\left(\mathbb{R}^{3}\right)\right) .
$$

Proof. To prove the uniqueness we consider two solutions $\mathbf{u}, \overline{\mathbf{u}} \in C^{1}\left([0, T) ; C^{2}\left(\mathbb{R}^{3}\right)\right) \cap C^{1}\left([0, T) ; H^{2}\left(\mathbb{R}^{3}\right)\right)$. Let their difference be $\rho=\mathbf{u}-\overline{\mathbf{u}}$, where $\rho=\left(\rho_{1}, \rho_{2}, \rho_{3}\right), \mathbf{u}=(u, v, w), \overline{\mathbf{u}}=(\bar{u}, \bar{v}, \bar{w})$. Then, subtracting the equations each other in (1), we have

$$
\left\{\begin{array}{l}
\rho_{t}=\rho \times \Delta \mathbf{u}+\overline{\mathbf{u}} \times \Delta \rho, \text { in } \Omega \times(0, T), \\
\rho(\mathbf{x}, 0)=(\mathbf{0}, 0), \text { in } \Omega, \\
\rho \mid \partial \Omega=(\mathbf{0}, 0) .
\end{array}\right.
$$

Multiplying the first equation of (37) by $-\Delta \rho$, integrating over $\Omega$, and using the Gauss formula, we obtain 


$$
\begin{gathered}
\left(\rho_{\mathrm{t}},-\Delta \rho\right)=\frac{1}{2} \frac{\mathrm{d}}{\mathrm{d} t}\|\nabla \rho\|_{L^{2}}^{2}, \\
\left(\rho_{\mathrm{t}},-\Delta \rho\right)=(\rho \times \Delta \mathbf{u}+\overline{\mathbf{u}} \times \Delta \rho,-\Delta \rho)=\int_{\Omega} \nabla \rho \cdot(\rho \times \Delta \nabla \mathbf{u}) \mathrm{d} \mathbf{x},
\end{gathered}
$$

for $\nabla \mathbf{u}=(\nabla u, \nabla v, \nabla w), \Delta \mathbf{u}=(\Delta u, \Delta v, \Delta w), \nabla \rho=\left(\nabla \rho_{1}, \nabla \rho_{2}, \nabla \rho_{3}\right)$.

By using $\mathbf{u}=\frac{1}{\sqrt{1+\left(c_{1} \mathrm{e}^{-\varepsilon} t+c_{2}\right)^{2}}}\left(\cos \theta, \sin \theta, c_{1} \mathrm{e}^{-\varepsilon} t+c_{2}\right), \quad \theta=\frac{c_{1} \mathrm{e}^{-2 \varepsilon}\left[(x-y)^{2}+(x-z)^{2}+(z-y)^{2}\right]}{12 \sqrt{1+\left(c_{1} \mathrm{e}^{-\varepsilon} t+c_{2}\right)^{2}}}$ in case 4, we obtain

$$
\nabla \mathbf{u}=\left(\begin{array}{ccc}
M_{2}(2 x-y-z) & M_{2}(2 y-x-z) & M_{2}(2 z-x-y) \\
N_{2}(2 x-y-z) & N_{2}(2 y-x-z) & N_{2}(2 z-x-y) \\
0 & 0 & 0
\end{array}\right)
$$

where $M_{2}=-\frac{\mathrm{e}^{-\varepsilon} c_{1} \sin \theta}{6\left(1+\left(c_{1} \mathrm{e}^{-\varepsilon} t+c_{2}\right)^{2}\right)}, N_{2}=-\frac{\mathrm{e}^{-\varepsilon} c_{1} \cos \theta}{6\left(1+\left(c_{1} \mathrm{e}^{-\varepsilon} t+c_{2}\right)^{2}\right)}$.

$$
\begin{aligned}
\|\Delta \nabla \mathbf{u}\|_{\infty} & =\sup \mid \frac{\mathrm{e}^{-5 \varepsilon} c_{1}^{3}}{36\left(1+\left(c_{1} \mathrm{e}^{-\varepsilon} t+c_{2}\right)^{2}\right)^{2}}\left[6\left(x^{3}+y^{3}+z^{3}\right)-9\left(x y^{2}-x z^{2}-z y^{2}-z x^{2}-y x^{2}-y z^{2}\right)+36 x y z\right] \\
& \leq\left|c_{4}(t)\right| .
\end{aligned}
$$

Inserting (41) into (39), it follows that

$$
\begin{aligned}
\frac{1}{2} \frac{\mathrm{d}}{\mathrm{d} t}\|\nabla \rho\|_{L^{2}}^{2} & =(\rho \times \Delta \mathbf{u}+\overline{\mathbf{u}} \times \Delta \rho,-\Delta \rho) \\
& \leq\|\Delta \nabla \mathbf{u}\|_{L^{\infty}}\|\nabla \rho\|_{L^{2}}^{2} \\
& \leq \mid c_{4}(t)\|\nabla \rho\|_{L^{2}}^{2} .
\end{aligned}
$$

Thanks to the Gronwall inequality, we have the following:

$$
\|\nabla \rho\|_{L^{2}}^{2} \leq \exp \left(\int_{0}^{t} 2\left|c_{4}(\tau)\right| \mathrm{d} \tau\right)\|\nabla \rho(\mathbf{x}, 0)\|_{L^{2}}^{2}=0,
$$

therefore we prove uniqueness of the solution in the sense of $C^{1}\left([0, T) ; C^{2}\left(\mathbb{R}^{3}\right)\right) \cap C^{1}\left([0, T) ; H^{2}\left(\mathbb{R}^{3}\right)\right)$.

\subsection{Stability}

In this section we discuss the stability of the solution, in $C^{\infty}(\Omega)$ and $L^{2}(\Omega)$ for the problem (1), respectively.

Let $\mathrm{u}=(u, v, w)$ is a solution of (1), where $\mathrm{u} \in C^{\infty} \cap H^{1}\left(\mathbb{R}^{3} \times[0, T)\right)$ from case $1, \overline{\mathrm{u}}=(\bar{u}, \bar{v}, \bar{w})$ denote the solution of a little disturbance, where $\mathrm{u} \in C^{\infty} \cap H^{1}\left(\mathbb{R}^{3} \times[0, T)\right)$. Let $\rho=\mathrm{u}-\overline{\mathrm{u}}$, be the difference of $\mathrm{u}$ and $\overline{\mathrm{u}}$, with initial value $\rho_{0}(x) \rightarrow 0$ in the sense of $L^{2}(\Omega)$ and boundary value $\mathrm{u} \rightarrow \overline{\mathrm{u}}$ in the sense of $L^{2}(\partial \Omega) \cap L^{\infty}(\partial \Omega), \forall t \in(0, T]$. Then, subtracting one equation from the other, we can get

$$
\left\{\begin{array}{l}
\rho_{t}=\rho \times \Delta \mathrm{u}+\overline{\mathrm{u}} \times \Delta \rho, \text { in } \Omega \times(0, T), \\
\rho(\mathrm{x}, 0)=\rho_{0}(x), \text { in } \Omega, \\
\rho \mid \partial \Omega=\varphi(x, t),
\end{array}\right.
$$

for $\Omega \in \mathbb{R}^{3}$ is a bounded domain. Multiplying this by $-\Delta \rho$, integrating over $\Omega$, and using the Gauss formula, 
we obtain

$$
\begin{gathered}
\left(\rho_{t},-\Delta \rho\right)=\frac{1}{2} \frac{\mathrm{d}}{\mathrm{d} t}\|\nabla \rho\|_{L^{2}}^{2}, \\
\left(\rho_{t},-\Delta \rho\right)=(\rho \times \Delta \mathrm{u}+\overline{\mathrm{u}} \times \Delta \rho,-\Delta \rho)=-\int_{\Omega} \rho \times \Delta \mathrm{u} \cdot \Delta \rho \mathrm{dx} \\
=-\int_{\partial \Omega} \nabla \rho \cdot(\rho \times \Delta \mathrm{u}) \cdot n \mathrm{ds}+\int_{\Omega} \nabla \rho \cdot \nabla(\rho \times \Delta \mathrm{u}) \mathrm{dx}, \\
\left|-\int_{\partial \Omega} \nabla \rho \cdot(\rho \times \Delta \mathrm{u}) \cdot n \mathrm{~d} s\right| \leq\|\Delta \mathrm{u}\|_{L^{\infty}(\partial \Omega)}\|\nabla \rho\|_{L^{2}(\partial \Omega)}\|\rho\|_{L^{2}(\partial \Omega)} \rightarrow 0, \\
\left|\int_{\Omega} \nabla \rho \cdot \nabla(\rho \times \Delta \mathrm{u}) \mathrm{dx}\right| \leq\|\Delta \nabla u\|_{L^{\infty}(\Omega)}\|\nabla \rho\|_{L^{2}(\Omega)}^{2} .
\end{gathered}
$$

By using $u(x, y, z, t)=k_{1} \mathrm{e}^{\varepsilon}(x+y+z), v(x, y, z, t)=k_{2} \mathrm{e}^{\varepsilon} \cos \frac{\mathrm{e}^{-\varepsilon}(x+y+z)}{3 k_{1}(T-t)}$

$w(x, y, z, t)=-k_{2} \mathrm{e}^{\varepsilon} \sin \frac{\mathrm{e}^{-\varepsilon}(x+y+z)}{3 k_{1}(T-t)}$ in case 1 , we obtain

$$
\begin{aligned}
\|\Delta \nabla \mathrm{u}\|_{\infty} & =\max \left\{3 \Delta k_{1} \mathrm{e}^{\varepsilon}, \Delta\left(-\frac{k_{2}}{k_{1}(T-t)} \sin \theta\right), \Delta\left(-\frac{k_{2}}{k_{1}(T-t)} \cos \theta\right)\right\} \\
& =\sup \left|\frac{\mathrm{e}^{-2 \varepsilon} k_{2}}{3 k_{1}^{3}(T-t)^{3}}\right| \leq\left|c_{2}(t)\right| .
\end{aligned}
$$

Hence

$$
\frac{\mathrm{d}}{\mathrm{d} t}\|\nabla \rho\|_{L^{2}(\Omega)}^{2} \leq 2\left|c_{2}(t)\right|\|\nabla \rho\|_{L^{2}(\Omega)}^{2}+2\|\Delta \mathrm{u}\|_{L^{\infty}(\partial \Omega)}\|\nabla \rho\|_{L^{2}(\partial \Omega)}\|\rho\|_{L^{2}(\partial \Omega)},
$$

since $\mathrm{u} \rightarrow \overline{\mathrm{u}}$ in the sense of $L^{2}(\partial \Omega) \cap L^{\infty}(\partial \Omega)$, we can make, for every given $\varepsilon>0$

$$
\|\Delta \mathrm{u}\|_{L^{\infty}(\partial \Omega)}\|\nabla \rho\|_{L^{2}(\partial \Omega)}\|\rho\|_{L^{2}(\partial \Omega)} \leq \varepsilon
$$

Using the Gronwall inequality in (44)-(47) for every $t \in(0, T)$ :

$\|\nabla \rho\|_{L^{2}}^{2} \leq \exp \left(\int_{0}^{t} 2\left|c_{2}(\tau)\right| \mathrm{d} \tau\right)\left[\left\|\nabla \rho_{0}(\mathrm{x}, 0)\right\|_{L^{2}}^{2}+2 \varepsilon \int_{0}^{t} \exp \left(-\int_{0}^{s} 2\left|c_{2}(\tau)\right| \mathrm{d} \tau\right) \mathrm{d} s\right] \rightarrow 0 \quad$ as $\quad \mathrm{u} \rightarrow \overline{\mathrm{u}}$ in the sense of $L^{2}(\partial \Omega) \cap L^{\infty}(\partial \Omega), \forall t \in(0, T]$ and $\left\|\rho_{0}(\mathrm{x}, 0)\right\|_{L^{2}}^{2} \rightarrow 0$. So we reach the stability of the solution in finite time.

In a similar way, by using

$$
\left\{\begin{array}{l}
\mathrm{u}(r, t)=\mathrm{e}^{4 \varepsilon}\left(k_{1} r^{-3}, k_{2} r^{-3} \cos \theta,-k_{2} r^{-3} \sin \theta\right), \\
\theta=-\frac{\mathrm{e}^{-5 \varepsilon} r^{5}}{25 k_{1}\left(T-\mathrm{e}^{-\varepsilon} t\right)},
\end{array}\right.
$$

in case 3 , and by using

$$
\left\{\begin{array}{l}
\mathrm{u}=\frac{1}{\sqrt{1+\left(c_{1} \mathrm{e}^{-\varepsilon} t+c_{2}\right)^{2}}}\left(\cos \theta, \sin \theta, c_{1} \mathrm{e}^{-\varepsilon} t+c_{2}\right), \\
\theta=\frac{c_{1} \mathrm{e}^{-2 \varepsilon}\left[(x-y)^{2}+(x-z)^{2}+(z-y)^{2}\right]}{12 \sqrt{1+\left(c_{1} \mathrm{e}^{-\varepsilon} t+c_{2}\right)^{2}}},
\end{array}\right.
$$

in case 4 , we can get the same conclusions. 


\section{Uniqueness and Stability of the Landau-Lifshitz-Gilbert Equation}

Because of the Landau-Lifshitz-Gilbert equation $\mathrm{u}_{t}=-\mathrm{u} \times \Delta \mathrm{u}-\lambda \mathrm{u} \times(\mathrm{u} \times \Delta \mathrm{u})$ in director fields $\mathrm{u}=(0, \infty) \times \mathbb{R}^{n} \rightarrow \mathbb{S}^{2}$ with values in the unit sphere $\mathbb{S}^{2} \subset \mathbb{R}^{3}$ where typically $n=3$, we find the Gilbert damping term $-\lambda \mathrm{u} \times(\mathrm{u} \times \Delta \mathrm{u})=\lambda\left(\Delta \mathrm{u}+|\nabla \mathrm{u}|^{2} \mathrm{u}\right)$, it would be easier and the stability of it has been done. We can see it in [20]. In this section, we study the uniqueness and stability of the initial boundary value problem for the Landau-Lifshitz-Gilbert equation below:

$$
\mathrm{u}_{t}=-\mathrm{u} \times \Delta \mathrm{u}-\lambda \mathrm{u} \times(\mathrm{u} \times \Delta, \mathrm{u}), \mathrm{u} \in \mathbb{R}^{3}, t \in \mathbb{R}^{+},
$$

observe that $\mathrm{u} \times(\mathrm{u} \times \Delta \mathrm{u})=(\mathrm{u} \cdot \Delta \mathrm{u}) \mathrm{u}-|\mathrm{u}|^{2} \Delta \mathrm{u}$, and we have the following results and it should be results that matter instead.

\subsection{Uniqueness}

Theorem 5. There exists $\Omega \in \mathbb{R}^{3}$ is a bounded domain. Then we the following initial value problem:

$$
\left\{\begin{array}{l}
\boldsymbol{v}_{t}=-\boldsymbol{v} \times \Delta \boldsymbol{v}-\lambda \boldsymbol{v} \times(\boldsymbol{v} \times \Delta \boldsymbol{v}), \boldsymbol{v} \in \mathbb{R}^{3}, t \in \mathbb{R}^{+}, \\
\boldsymbol{v}_{0}=A \boldsymbol{v}_{0}=A \boldsymbol{u}(x, y, z, 0) \in H^{1}\left(\mathbb{R}^{3}\right), \\
\boldsymbol{v}=A \boldsymbol{u}(x, y, z, t), \text { on } \partial \Omega \times[0, T),
\end{array}\right.
$$

has a unique smooth solution $\boldsymbol{v}=A \boldsymbol{u} \in H^{1}\left(\mathbb{R}^{3} \times[0, T)\right)$.

Proof. Let their difference be $\rho=\mathbf{v}-\overline{\mathbf{v}}$, where $\rho=\left(\rho_{1}, \rho_{2}, \rho_{3}\right), \mathbf{v}=A \mathbf{u}=A(u, v, w) \in H^{1}\left(\mathbb{R}^{3} \times[0, T)\right)$, $\overline{\mathbf{v}}=A \overline{\mathbf{u}}=A(\bar{u}, \bar{v}, \bar{w}) \in H^{1}\left(\mathbb{R}^{3} \times[0, T)\right)$. Then, subtracting the equations each other in (52), we have

$$
\left\{\begin{array}{l}
\rho_{t}=-\mathbf{v} \times \Delta \mathbf{v}+\overline{\mathbf{v}} \times \Delta \overline{\mathbf{v}}-\lambda[\mathbf{v} \times(\mathbf{v} \times \Delta \mathbf{v})-\overline{\mathbf{v}} \times(\overline{\mathbf{v}} \times \Delta \overline{\mathbf{v}})], \Omega \times(0, T), \\
\rho(\mathbf{x}, 0)=(\mathbf{0}, 0), \text { in } \Omega, \\
\rho \mid \partial \Omega=(\mathbf{0}, 0),
\end{array}\right.
$$

we obtain that if $\mathbf{v}, \overline{\mathbf{v}}$ are known solutions, we can get $\mathbf{v}, \overline{\mathbf{v}} \in L^{\infty}\left((0, T) ; \mathbb{R}^{n}\right)$. Multiplying the first equation of (53) by $-\Delta \rho$, integrating over $\Omega$, and using the Gauss formula, we obtain

$$
\begin{aligned}
& \frac{1}{2} \frac{\mathrm{d}}{\mathrm{d} t}\|\nabla \rho\|_{L^{2}}^{2} \\
& =\int_{\Omega}(\mathbf{v} \times \Delta \mathbf{v}+\overline{\mathbf{v}} \times \Delta \rho-\overline{\mathbf{v}} \times \Delta \mathbf{v}) \cdot \Delta \rho+\lambda[\mathbf{v} \times(\mathbf{v} \times \Delta \mathbf{v})+\overline{\mathbf{v}} \times(\overline{\mathbf{v}} \times \Delta \rho)-\overline{\mathbf{v}} \times(\overline{\mathbf{v}} \times \Delta \mathbf{v})] \cdot \Delta \rho \mathrm{d} \mathbf{x} \\
& =\int_{\Omega}(\rho \times \Delta \mathbf{v}) \cdot \Delta \rho+\lambda[\mathbf{v} \times(\rho \times \Delta \mathbf{v})+\overline{\mathbf{v}} \times(\overline{\mathbf{v}} \times \Delta \rho)+\rho \times(\overline{\mathbf{v}} \times \Delta \mathbf{v})] \cdot \Delta \rho \mathrm{d} \mathbf{x} \\
& \leq \int_{\Omega}(\rho \times \Delta \mathbf{v}) \cdot \Delta \rho+\lambda[\mathbf{v} \times(\rho \times \Delta \mathbf{v})+\rho \times(\overline{\mathbf{v}} \times \Delta \mathbf{v})] \cdot \Delta \rho \mathrm{d} \mathbf{x} \\
& \leq\|\Delta \mathbf{v}\|_{L^{\infty}(\Omega)}\|\nabla \rho\|_{L^{\infty}(\Omega)}^{2}+\lambda\|\Delta \mathbf{v}\|_{L^{\infty}(\Omega)}\|\nabla \rho\|_{L^{\infty}(\Omega)}^{2}+\|\overline{\mathbf{v}}\|_{L^{\infty}(\Omega)}\|\Delta \mathbf{v}\|_{L^{\infty}(\Omega)}\|\nabla \rho\|_{L^{\infty}(\Omega)}^{2} \\
& \leq\left.(1+\lambda+\bar{C}(t))\right|_{C}(t) \mid\|\nabla \rho\|_{L^{\infty}(\Omega)}^{2} .
\end{aligned}
$$

As

$$
\Delta \rho \cdot[\overline{\mathbf{v}} \times(\overline{\mathbf{v}} \times \Delta \rho)]=\Delta \rho \cdot\left[(\overline{\mathbf{v}} \cdot \Delta \rho) \overline{\mathbf{v}}-|\overline{\mathbf{v}}|^{2} \Delta \rho\right]=(\Delta \rho \cdot \overline{\mathbf{v}})^{2}-|\overline{\mathbf{v}}|^{2}|\Delta \rho|^{2} \leq 0,
$$

for $\nabla \mathbf{v}=A \nabla \mathbf{u}=A(\nabla u, \nabla v, \nabla w), \Delta \mathbf{v}=A \Delta \mathbf{u}=A(\Delta u, \Delta v, \Delta w), \nabla \rho=\left(\nabla \rho_{1}, \nabla \rho_{2}, \nabla \rho_{3}\right)$.

Thanks to the Gronwall inequality, we have the following:

$$
\|\nabla \rho\|_{L^{2}}^{2} \leq \exp \left(\int_{0}^{t} 2|c(\tau)|(1+\lambda+\bar{c}(\tau) \mathrm{d} \tau)\right)\|\nabla \rho(\mathbf{x}, 0)\|_{L^{2}}^{2}=0,
$$

therefore we can prove the uniqueness of the solution in the sense of $H^{1}\left(\mathbb{R}^{3}\right), \forall 0 \leq t \leq T$.

\subsection{Stability}

In this section we discuss the stability of the solution, in $C^{2}(\Omega)$ and $L^{2}(\Omega)$ for the problem (51), respec- 
tively.

Assume $\mathrm{v}=A \mathrm{u}=A(u, v, w)$, where $\mathrm{v} \in H^{1}\left(\mathbb{R}^{3} \times(0, T)\right)$. Let $\overline{\mathrm{v}}=A \overline{\mathrm{v}}=A(\bar{u}, \bar{v}, \bar{w})$ denote the solution of a little disturbance, where $\overline{\mathrm{v}} \in H^{1}\left(\mathbb{R}^{3} \times(0, T)\right)$. Let $\rho=\mathrm{v}-\overline{\mathrm{v}}$ be different of $\mathrm{v}, \overline{\mathrm{v}}$, with initial value $\rho_{0}(x) \rightarrow 0$ in the sense of $L^{2}(\Omega)$ and boundary value $\mathrm{v} \rightarrow \overline{\mathrm{v}}$ in the sense of

$L^{2}(\partial \Omega) \cap L^{\infty}(\partial \Omega), \forall t \in(0, T]$. Then, subtracting one equation from the other, we can get

$$
\left\{\begin{array}{l}
\rho_{t}=-\mathrm{v} \times \Delta \mathrm{v}+\overline{\mathrm{v}} \times \Delta \overline{\mathrm{v}}-\lambda[\mathrm{v} \times(\mathrm{v} \times \Delta \mathrm{v})-\overline{\mathrm{v}} \times(\overline{\mathrm{v}} \times \Delta \overline{\mathrm{v}})], \Omega \times(0, T), \\
\rho(\mathrm{x}, 0)=(0,0), \text { in } \Omega, \\
\rho \mid \partial \Omega=\varphi(x, t),
\end{array}\right.
$$

for $\Omega \in \mathbb{R}^{3}$ is a bounded domain. Multiplying this by $-\Delta \rho$, integrating over $\Omega$, and using the Gauss formula, we obtain

$$
\begin{aligned}
& \frac{1}{2} \frac{\mathrm{d}}{\mathrm{d} t}\|\nabla \rho\|_{L^{2}}^{2} \\
& =\int_{\Omega}(\mathrm{v} \times \Delta \mathrm{v}+\overline{\mathrm{v}} \times \Delta \rho-\overline{\mathrm{v}} \times \Delta \mathrm{v}) \cdot \Delta \rho+\lambda[\mathrm{v} \times(\mathrm{v} \times \Delta v)+\overline{\mathrm{v}} \times(\overline{\mathrm{v}} \times \Delta \rho)-\overline{\mathrm{v}} \times(\overline{\mathrm{v}} \times \Delta \mathrm{v})] \cdot \Delta \rho \mathrm{dx} \\
& =\int_{\Omega}(\rho \times \Delta \mathrm{v}) \cdot \Delta \rho+\lambda[\mathrm{v} \times(\rho \times \Delta \mathrm{v})+\overline{\mathrm{v}} \times(\overline{\mathrm{v}} \times \Delta \rho)+\rho \times(\overline{\mathrm{v}} \times \Delta \mathrm{v})] \cdot \Delta \rho \mathrm{dx} \\
& \leq \int_{\Omega}(\rho \times \Delta \mathrm{v}) \cdot \Delta \rho+\lambda[\mathrm{v} \times(\rho \times \Delta \mathrm{v})+\rho \times(\overline{\mathrm{v}} \times \Delta \mathrm{v})] \cdot \Delta \rho \mathrm{dx} \\
& \leq\|\Delta v\|_{L^{\infty}(\Omega)}\|\nabla \rho\|_{L^{\infty}(\Omega)}^{2}+\lambda\|\Delta v\|_{L^{\infty}(\Omega)}\|\nabla \rho\|_{L^{\infty}(\Omega)}^{2}+\|\bar{v}\|_{L^{\infty}(\Omega)}\|\Delta v\|_{L^{\infty}(\Omega)}\|\nabla \rho\|_{L^{\infty}(\Omega)}^{2} \\
& +\left(\|\Delta v\|_{L^{\infty}(\Omega)}+\|v\|_{L^{\infty}(\partial \Omega)}\|\Delta v\|_{L^{\infty}(\partial \Omega)}+\|\bar{v}\|_{L^{\infty}(\partial \Omega)}\|\Delta v\|_{L^{\infty}(\partial \Omega)}\right)\|\nabla \rho\|_{L^{2}(\partial \Omega)}\|\rho\|_{L^{2}(\partial \Omega)} \\
& \leq(1+\lambda+\bar{C}(t)) \mid c(t)\|\nabla \rho\|_{L^{\infty}(\Omega)}^{2}+\varepsilon,
\end{aligned}
$$

since $\mathrm{v} \rightarrow \overline{\mathrm{v}}$ in the sense of $L^{2}(\partial \Omega) \cap L^{\infty}(\partial \Omega)$, we can make, for every given $\varepsilon>0$ :

$$
\left(\|\Delta \mathrm{v}\|_{L^{\infty}(\partial \Omega)}+\|\mathrm{v}\|_{L^{\infty}(\partial \Omega)}\|\Delta \mathrm{v}\|_{L^{\infty}(\partial \Omega)}+\|\overline{\mathrm{v}}\|_{L^{\infty}(\partial \Omega)}\|\Delta \mathrm{v}\|_{L^{\infty}(\partial \Omega)}\right)\|\nabla \rho\|_{L^{2}(\partial \Omega)}\|\rho\|_{L^{2}(\partial \Omega)} \leq \varepsilon
$$

where $\nabla \mathrm{v}=A \nabla \mathrm{u}=A(\nabla u, \nabla v, \nabla w), \Delta \mathrm{v}=A \Delta \mathrm{u}=A(\Delta u, \Delta v, \Delta w), \nabla \rho=\left(\nabla \rho_{1}, \nabla \rho_{2}, \nabla \rho_{3}\right)$.

Thanks to the Gronwall inequality, we have the following:

$$
\begin{aligned}
\|\nabla \rho\|_{L^{2}}^{2} \leq & \exp \left(\int_{0}^{t} 2|c(\tau)|(1+\lambda+\bar{c}(\tau) \mathrm{d} \tau)\right)\|\nabla \rho(\mathrm{x}, 0)\|_{L^{2}}^{2} \\
& +2 \varepsilon \int_{0}^{t} \exp \left(-\int_{0}^{s} 2|c(\tau)|(1+\lambda+\bar{c}(\tau)) \mathrm{d} \tau\right) \mathrm{d} s \rightarrow 0,
\end{aligned}
$$

therefore we prove the stability of the solution in the sense of $H^{1}\left(\mathbb{R}^{3} \times(0, T)\right)$.

\section{Conclusion}

In this paper, we study the symmetry reductions and explicit solutions by means of classical Lie group method. First, we get the infinitesimal generator and group invariant solutions to multidimensional Landau-Lifshitz equation. Then, we build the relations between new solutions and olds have been found. Finally, via these explicit solutions,we study the uniqueness and stability of initial-boundary problem on multidimensional LandauLifshitz equation.

\section{Acknowledgements}

This work was supported by the Natural Foundation of China (No. 11561076, No. 11101356).

\section{References}

[1] Landau, L.D. and Lifshitz, E.M. (1935) On the Theory of the Dispersion of Magnetic Permeability Inferroagnetic Bodies. Phys. Z. Sowj, 8, 153-169. (Reproduced in Collected Papers of Landau L.D., Pergamon Press, New York, 1965, pp. 101-114.) 
[2] Conte, R. and Chow, K.W. (2008) Doubly Periodic Waves of a Discrete Nonlinear Schröndinger System with Saturable Nonlinearity. Journal of Nonlinear Mathematical Physics, 15, 398-409. http://dx.doi.org/10.2991/jnmp.2008.15.4.4

[3] Zhou, Y. and Guo, B.L. (1986) The Weak Solution of Honmogeneons Boundary Value Problem for the System of Ferromagnetic Chain with Several Variables. Seientia Sinica A, 4, 337-349.

[4] Chang, N.H., Shatah, J. and Ulenbeck, K. (2000) Schröndinger Maps. Communications on Pure and Applied Mathematics, 53, 590-602.

[5] Lakshmanan, M., Ruijgrok, T.W. and Thompson, C.J. (1976) On the Dynamics of a Continuum Spin System. Physiea A, 84, 577-590. http://dx.doi.org/10.1016/0378-4371(76)90106-0

[6] Nakamura, K. and Sasada, T. (1974) Solition and Wave Trains in Ferromagnets. Physics Letters A, 48, $321-322$. http://dx.doi.org/10.1016/0375-9601(74)90447-2

[7] Tjof, J. and Wright, J. (1977) Soliton in the Hesenberg Chain. Physical Review E, 15, 3470-3476.

[8] Guo, B.L. and Yang, G.S. (2001) Some Exact Nontrivial Global Solutions with Values in Unit Sphere for Two-Dimensional Landau-Lifshitz Equations. Journal of Mathematical Physics, 42, 5223-5227. http://dx.doi.org/10.1063/1.1402955

[9] Guo, B.L., Han, Y.Q. and Yang, G.S. (2000) Blow up Problem for Landau-Lifshitz Equations in Two Dimensions. Communications in Nonlinear Science and Numerical Simulation, 5, 43-44. http://dx.doi.org/10.1016/S1007-5704(00)90023-6

[10] Guo, B.L., Han, Y.Q. and Yang, G.S. (2001) Exact Blow-Up Solutions for Multidimensional Landau-Lifshitz Equations. Advances in Mathematics, 30, 91-93.

[11] Yang, G.S. and Chang, Q.S. (2003) Limit Behavior of Solution for Multidimensional Landau-Lifshitz Equations with External Magnetic Field. Physics Letters A, 318, 270-280. http://dx.doi.org/10.1016/j.physleta.2003.08.059

[12] Yang, G.S., Zhang, Y.Z. and Liu, L.M. (2009) Explicit Piecewise Smooth Solutions of Multidimensional Landau-Lifshitz Equation with Discontinuous External Field. Acta Mathematieae Applicatae Siniea, 25, $29-42$.

[13] Song, W.J. and Yang, G.S. (2014) Nonhomogeneous Boundary Value Problem for (I,J) Similar Solutions of Incompressible Two-Dimensional Euler Equations. Journal of Inequalities and Applications, 2014, 277-292. http://dx.doi.org/10.1186/1029-242X-2014-277

[14] Yang, G.S. (2011) Spherical Cone Symmetric Families Generated by Landau-Lifshitz Equation and Their Evolution. Scientia Sinica Mathematica, 41, 181-196. http://dx.doi.org/10.1360/012010-582

[15] Ovsiannikov, L.V. (1982) Group Analysis of Differential Equations.

[16] Tian, C. (2001) Lie Group and Its Applications to Differential Equations. Science Press, Beijing.

[17] Olver, P.J. (2000) Applications of Lie Group to Differential Equations. Springer Verlag, Berlin.

[18] Bluman, G.W. and Anco, S.C. (2002) Symmetry and Integration Methods for Differential Equations. Springer, New York.

[19] Xu, B. and Liu, X.Q. (2001) Group Invariant Solutions and Conservation Laws of Landau-Lifshitz Equation. Acta Mathematieae Applicatae Siniea, 40, 575-579.

[20] Christof, M. (2011) Global Solvability of the Cauchy Problem for the Landau-Lifshitz-Gilbert Equation in Higher Dimensions. Indiana University Mathematics Journal, 61, 1175-1200.

[21] Fan, E. (2000) Extended Tanh-Function Method and Its Applications to Nonlinear Equations. Physics Letters A, 277, 212-218. http://dx.doi.org/10.1016/S0375-9601(00)00725-8

[22] Bluman, G.W. and Cole, J.D. (1974) Similarity Methods for Differential Equations. Springer, New York.

[23] Evance, L.C. (2002) Partial Differential Equations. American Mathematical Society, Providence. 